\title{
Determination of Data Quality and Results from two Mount Hopkins Robotic Telescopes
}

\author{
Diane M. Pyper ${ }^{1}$, S.J. Adelman², R.J. Dukes, Jr. ${ }^{3}$, G.P. McCook ${ }^{4}$, \\ M.A. Seeds ${ }^{5}$ \\ ${ }^{1}$ University of Nevada-Las Vegas, USA, ${ }^{2}$ The Citadel, USA, ${ }^{3}$ College of \\ Charlestown, USA, ${ }^{4}$ Villanova University, USA, ${ }^{5}$ Franklin and Marshall College, \\ USA
}

\begin{abstract}
We present various methods for determining night quality from observations obtained with totally unattended automatic photoelectric telescopes at the Fairborn Observatory site on Mt. Hopkins, AZ. Telescopes are the Phoenix 10-inch (P10) and the Four College 75-cm (CAPT). Filter systems used are Johnson UBV, KronCousins RI and Strömgren uvby. As a preliminary data filter for the P10 "rent-astar" data, all observations with standard errors of the mean (SEM) above 20 mmag are discarded; a summary of nightly SEM's, number of aborts and total observing time is sent to each user. The CAPT data are evaluated by standard deviation of magnitudes or counts for photometric groups and for an entire night. The Geneva photometric statistics are computed for these latter data and have proved useful as a preliminary screen for bad data. Various criteria for good and bad groups and nights are discussed. Scientific results are presented for several magnetic CP stars that were observed using both telescopes. Differences in precision of the data and strategies for observing are discussed.
\end{abstract}

\section{Introduction}

Russ Genet has just given a very thorough description of the Mt. Hopkins robotic telescopes (this volume). I would like to describe the data taking process, evaluation and some results from two of these telescopes, the Phoenix 10-inch (P10) and the Four College 75 -cm (CAPT).

The P10, for which Mike Seeds is the Principal Astronomer (PA), carries out a basic program of Johnson UBV differential photometry for a large number of users on a "renta-star" basis. Each group, consisting of variable, comparison and check star, is observed once per night; check stars are observed twice and comparison stars four times per group. Reductions are made using average extinction and transformation coefficients determined on several standard star nights per observing season.

The CAPT is able to carry out a much more versatile program, as it uses the Automatic Telescope Instruction Set (ATIS) (Genet and Hayes 1989), which provides many more options for observing. At present the Four College users are four of the authors; Pyper, Dukes (PA), Adelman and McCook; plus Ed Guinan at Villanova. We are observing a 
wide variety of variable stars using Johnson UBV, Kron-Cousins RI, Strömgren fourcolor, $\beta$, and $\mathrm{H} \alpha$ systems. A group can be repeated many times a night and all-sky observations of standards and program stars can also be made. For every differential photometry group, at least two check star and four comparison star measurements are made per group. At present, we also use average coefficients for reductions.

The basic problems in evaluating these data are that there is no on-site evaluation of atmospheric conditions and instrument performance and no real-time interaction while the observations are being carried out. Therefore, the quality of the data must be evaluated after the observations are completed.

\section{Data Evaluation - P10}

As is the case for many photometric observers, we find the scatter and standard error of the mean (SEM) of individual observations of comparison and check stars within a group to be a useful criterion for the evaluation of a night. For the P10, SEM's are calculated for all stars, variables, comparison and check stars and the data for any star in a given filter are discarded if the SEM 20 mmag. Each quarter, the user receives a data file containing his/her data for that quarter, and a night report file for the P10. The night report file contains information about the quality of individual nights, including the length of time the roof was open in hours, the total number observations deleted by the 0.02 mag. filter, total number of observations made during the night, and the average and median SEM for the night. There is also a mountain log file which contains information about roof closings (clouds, rain) and data for the P10 concerning the number of starts, stops and number of groups aborted. The Rent-A-Star philosophy holds that users must be given all potentially useful data. The user is the only person who can decide which data are most useful in a given case. In judging the quality of the night, the user examines the night report, looking at all the information provided. A good night will be many hours long; will include many observations with only a few rejected by the 20 mmag filter; and the average and median SEM will be low. A bad night will be short, perhaps only a few hours, and may include only a few observations that were not rejected; the SEMs would probably be quite high. Some nights may be many hours long, but will contain few observations, a signal that the telescope spent a large part of the night closed, probably because of clouds. A long night with many observations made but few accepted by the 20 mmag filter was probably a poor night with scattered haze or clouds; the SEMs are typically high. Most troublesome are long nights with many observations and SEMs slightly higher than normal, which are proabably clear nights which were not quite photometric. The mountain log files give further clues. If the roof closed due to rain or thick clouds, this is stated in the log but the number of stops and starts and group aborts for the P10 should also be examined. On a good night, the telescope will have only one start at the beginning of the night and one stop at the end. Multiple starts and stops signal a night broken by clouds not quite thick enough to cause the roof to close. A high number of aborts is also a warning that the night may have been poor. An abort commonly occurs when the telescope is unable to locate the next star in the sequence, and such aborts can again be caused by clouds. 
Ultimately the evaluation of P10 data is left to the user. The $20 \mathrm{mmag}$ filter is designed to reject only the most serious errors such as those caused by twilight. All other data are sent to the user. Users observing large amplitude stars may need little further filtering, but users watching low amplitude stars will need to examine their data more carefully. Some users reject any data from incomplete groups. Because $U$ is so sensitive to sky conditions, it often has SEMs over $20 \mathrm{mmag}$ and the $U$ data is missing from a group. On less than photometric nights, groups may be missing data from other filters as well. By rejecting all such groups, the P10 users report precision of 5 mmags or better. While this certainly throws out some good data with the bad, it assures the highest quality. For example, the P10 data for CU Vir discussed below in Section 4 was pre-filtered to eliminate all groups with missing data. These data were further filtered to remove all observations with SEMs 10 mmag, leaving 626 observations in three observing seasons. This example further illustrates one of the big advantages of robotic telescopes; an abundance of data. With observing programs of variable stars on conventional telescopes, the amount of data is ususally sparse and takes many years to collect, thus the observer is tempted to retain any data that possibly may be useful and probably keeps much marginal data that should be discarded. Even though old habits are hard to break, it is a lot easier to discard questionable data when one knows there is a large body of better data available.

\section{Data Evaluation - CAPT}

With the CAPT data, ATIS permits further options for the evaluation of night quality. It is possible for each user to examine the SEM and residuals of each comparison and check star observed during a night (either as magnitudes or as a percentage of the mean photon count). McCook (1991) has written software that does this. An initial look at a plot of residuals vs time or SEMs vs time gives a good idea of what the night was like and examination of such plots can immediately eliminate terrible nights. ATIS also provides the user with detailed information concerning the reasons for group aborts, such as "star not found", "too near moon", "outside of observing window", etc. As with the P10, a check for nights with many "star not found" aborts, small numbers of groups observed, etc. can flag poor nights. A further screening process is made possible by the fact that each integration interval consists of a number of $100 \mathrm{~ms}$ sub-intervals in order to generate the Geneva Statistics (Bartholdi et al. 1984). The telescope output for each observation thus includes the total count, $\mathrm{N}=\mathrm{N}_{\mathrm{i}}$, where $\mathrm{N}_{\mathrm{i}}$ is the count in a given sub-interval, plus the Geneva statistics $Q, R$, and $G$. Within the total integration interval, $Q$ is sensitive to scintillation, $R$ to oscillation and drift and $G$ to sharp spikes and drops. We have found (McCook 1992) that the R statistic correlates fairly well with sky conditions, but simply removing readings with bad statistics will not insure high quality data. However, we have found the Geneva statistics to be useful as the next step in the filtering process, which is to remove all data with $\mathrm{Q}>10, \mathrm{R}>1.5$ or $<0.5$, and $\mathrm{G}>0.006$ or $<-0.006$ ( $\mathrm{G}$ seems to be only sensitive to instrumental problems).

The SEMs are clearly the best indicator of sky and data quality and can be used with confidence to identify and eliminate questionable data from robotic telescopes. Each of the CAPT users has his/her own favorite method of deciding which data to finally keep. 
One way of evaluating the data (used by DMP) is to calculate SEMs on the photon counts for each constant star and to express these as percentages of the mean counts. If any constant star in a group has an SEM $>2 \%$, it is called a bad group. The number of bad groups in a night's observation is then counted and that number is expressed as a percentage of the total number of groups $(=\% \mathrm{BG})$ successfully observed that night (i.e., groups that have survived the previous filtering processes). Any night with $\% \mathrm{BG}>90 \%$ can be eliminated immediately; those with \%BG between $70 \%$ and $90 \%$ are usually no good, but individual groups are examined anyway. On all the other nights, all of the bad groups are examined; the decision to keep or discard the data is based on the program and the individual variable star (most of such data are usually discarded).

\section{Some Scientific Results for Upper Main Sequence Chemically Peculiar Stars}

As mentioned previously, robotic telescopes present great advantages for the observer of variable stars. A much greater amount of data can be collected compared to conventional telescopes, as the observer's presence is not required; time-sharing in a given night by a number of different observers is also advantageous as this results in greater phase coverage for a given variable. On the CAPT, short-period variables and eclipsing binaries can be observed in long runs over one night or several nights as well. The observer is also able to collect data over a period of several years, giving him/her a homogeneous data set for year-to-year comparisons.

Because of these advantages, two of the authors (DMP and SJA) have begun studies to search for changes in the shapes of the light curves of the two magnetic chemically peculiar (MCP) stars with the shortest known periods. CU Vir and 56 Ari have periods of about 0.52 days and 0.73 days, respectively. Light, spectrum and magnetic variations in MCP stars are believed to be due to rotation and inhomogeneous atmospheres with large magnetic fields. Shore and Adelman (1976) suggested that MCP stars that were not in binary systems could experience free body precession due to a distortion in the shape of the star by the magnetic field. Since the precession period would be many times that of the rotation period of the star, the shortest period variables are the most likely candidates to display changes in their light curves due to precession. For stars with periods less than one day, the precession periods would be expected to be about 5 to 10 years. Adelman and Fried (1992) have found changes in the shape of the UBV light curves of 56 Ari compared with earlier published data. Two years of CAPT uvby data obtained by Adelman may also show some changes in the shapes of the $u$ and $v$ light curves, although the results are preliminary .

Three years of P10 UBV data for CU Vir were studied by DMP and compared with UBV data of Hardie (1958). An improved period of 0.5206800 days (Adelman et al. 1992) was used. There is some evidence for changes in shape between the Hardie and P10 data, although this is not compelling due to phase gaps in the Hardie data. The three years of the P10 data were separately compared. There appear to be subtle differences from year to year, the principal of which are a slight change in the time of minimum for the 1989 data with respect to the 1987 and 1988 data; and systematic changes in average brightness and perhaps amplitude for the $U$ data from year to year (Figure 1). It is clear 
that higher precision is necessary in order to further pursue such studies. Some CAPT uvby data are available for CU Vir in 1991-92 but are not sufficient to come to any conclusions about yearly changes in the light curves; the star is on the program for the next few years including some long nightly runs to check on cycle-to-cycle variations. Additionally, there is difficulty in fitting all the available data for $\mathrm{CU}$ Vir to a single period, a problem which will be further investigated in the future.

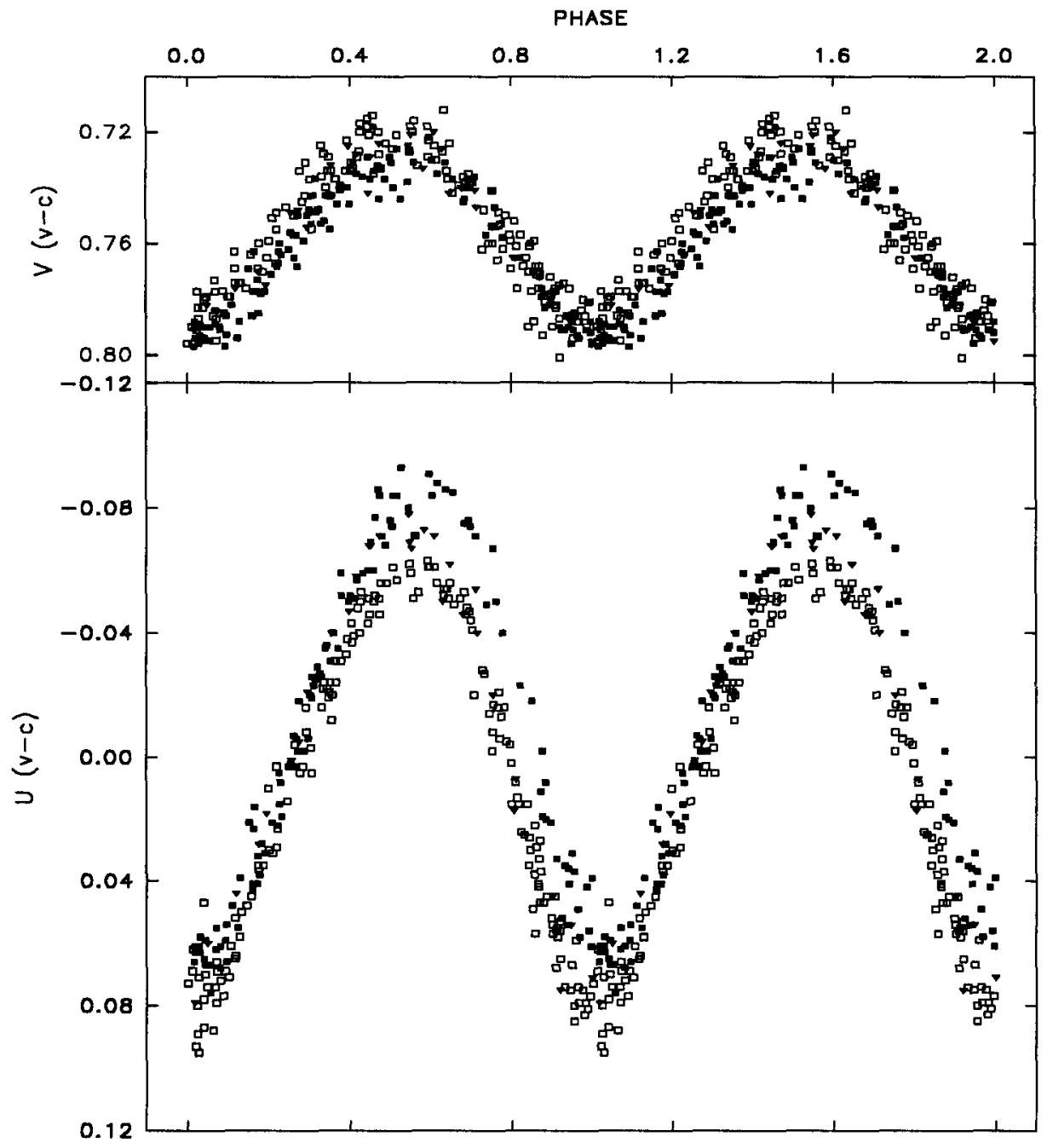

Figure $1 \mathrm{~V}$ and U photometry for CU Vir from P10 observations. Filled inverted triangles, open squares and filled squares are 1987, 1988 and 1989 data, respectively. 


\section{Future Improvements}

The CAPT group will carry out two major projects in the next year or so to improve both the quality of our data and our observing efficiency. The first of these is to incorporate observations of standard stars into the nightly observations rather than relying on standard star nights and average extinction and transformation coefficients. We also plan to use a scheme to incorporate all the constant stars into the characterization of a night or a run of nights, similar to that described by Manfroid and Heck (1983).

Secondly, as mentioned above, we schedule on a time-share basis, rather than granting blocks of time to a single observer. Because of this, we need a much more sophisticated way to plan observations than we have at present. Even with our five regular observers plus students and collegues who need occasional observations, the scheduling procedure becomes very complicated for the PA, especially since he must insure that each school gets its fair share of telescope time. Additionally, we are now receiving an increasing number of requests for observing time by observers organizing international campaigns and those who have groups of stars they would like observed. Mark Drummond and others at NASA/Ames is writing scheduling software based on artificial intelligence for robotic telescopes with an eye toward future networks of such telescopes and possible lunar-based networks. The CAPT group hopes to be an early "guinea pig" for this software; we feel that a system such as this will be essential for efficient operation of multi-user robotic telescopes.

\section{References:}

Adelman, S.J. and Fried, R., 1992, in preparation.

Adelman, S.J., Dukes, Jr., R.J., Pyper, D.M., 1992, Astron. J., Astron. J., 104, in press.

Bartholdi, P., Burnet, M., Rufener, F., 1984, Astron. Astrophys. 134, 290.

Genet, R.M. and Hayes, D.S., 1989, Robotic Observatories, AutoScope Corp., Mesa, AZ, p. 28.

Hardie, R., 1958, Astrophys. J, 127, 620.

Manfroid, J. and Heck, A., 1983, Astron. Astrophys. 120, 302.

McCook, G.P., 1991, in: Advances in Robotic Telescopes, ed. M. Seeds, Fairborn Press, Mesa, AZ, p. 263.

McCook, G.P., 1992, in: Robotic Telescopes in the 1990s, ed. A. Filippenko, Astr. Soc. of Pacific Conf. Series, in press.

Shore, S.N. and Adelman, S.J., 1976, Astrophys. J. 120,816. 


\section{Discussion}

T.J. Kreidl: To what accuracy is the time actually recorded for each data point?

Pyper: We get fractional JD to six decimal points, or about 0.1 sec.

E. F. Milone: I noticed that the check and comparison stars are observed at different times in the 'group'. It would be better to have an extra comparison star observation at each end of the sequence to get the same mean time for it as the check star observations.

Pyper: The P10 sequence is "set in stone" but ATIS enables us to vary our sequences, so we now mostly do follow your suggested sequence. 